\title{
CONVEX FUNCTIONS WITH RESTRICTED CURVATURE
}

\author{
D. STYER AND D. J. WRIGHT
}

(Communicated by Clifford J. Earle, Jr.)

\begin{abstract}
Given $0 \leq R_{1} \leq R_{2} \leq \infty$, we consider a class of normalized convex functions $f$ in the unit disk $\mathbf{D}$, for which $\partial f(\mathbf{D})$ satisfies a Blaschke Rolling Theorem condition with radii $R_{1}$ and $R_{2}$. This class contains the convex functions of bounded type. We study the geometry of the image region $f(\mathbf{D})$ and various covering and distortion properties.
\end{abstract}

\section{INTRODUCTION}

Let $B(a, \delta)$ be the open disk of radius $\delta$ centered at $a$, and let $\mathbf{D}=$ $B(0,1)$. As usual, $\mathbf{S}$ denotes the class of normalized univalent functions in $\mathbf{D}$, and we denote by $\mathrm{CV}$ the subclass of $\mathbf{S}$ consisting of convex functions. Assume $f \in \mathrm{CV}$. For fixed $r \in(0,1)$ the curve $C_{r}: w=f\left(r e^{i t}\right), 0 \leq t \leq 2 \pi$, has radius of curvature at $f(z)$ given by

$$
\rho(z)=\left|z f^{\prime}(z)\right| / \operatorname{Re}\left\{1+z f^{\prime \prime}(z) / f^{\prime}(z)\right\} .
$$

When clarity requires reference to the function $f$ we will write $\rho(f ; z)$, and we adopt a similar convention when it is needed in notations which arise later. Let $\rho_{M}(r)$ and $\rho_{m}(r)$ denote the maximum and minimum, respectively, of $\rho(z)$ on $|z|=r$. Setting $n(z)=z f^{\prime}(z) /\left|z f^{\prime}(z)\right|$ (the unit exterior normal to $C_{r}$ at $\left.f(z)\right)$, Blaschke's Rolling Theorem (e.g., see [3]) implies that

$$
B\left(f(z)-\rho_{m}(r) n(z), \rho_{m}(r)\right) \subseteq f(B(0, r)) \subseteq B\left(f(z)-\rho_{M}(r) n(z), \rho_{M}(r)\right)
$$

for each $z$ with $|z|=r$.

Walsh [8] showed that either $\rho_{M}(r) / r$ is strictly increasing on $(0,1)$ or $f(z)=z$. He also observed that $\rho_{m}(r)$ need not be monotone. Let

$$
R_{2}(f)=\lim _{r \rightarrow 1} \rho_{M}(r)
$$

and

$$
R_{1}(f)=\liminf _{r \rightarrow 1} \rho_{m}(r) .
$$

Since $\rho(z) /|z| \rightarrow\left|f^{\prime}(0)\right|=1$ as $z \rightarrow 0$, we see from Walsh's result that $1 \leq$ $\rho_{M}(r) / r \leq R_{2}(f)$, with equality if and only if $f(z)=z$. For $0 \leq R_{1} \leq R_{2} \leq \infty$

Received by the editors January $17,1989$.

1980 Mathematics Subject Classification (1985 Revision). Primary 30C45. 
and $R_{2} \geq 1$ let $\mathrm{CV}\left(R_{1}, R_{2}\right)=\left\{f \in \mathrm{CV}: R_{1} \leq R_{1}(f) \leq R_{2}(f) \leq R_{2}\right\}$. These functions were studied by Goodman [1,2], who called them convex functions of bounded type. Distortion and coefficient results for this class have been obtained by Wirths [9] and Mejia and Minda [4].

In this paper we consider a closely related class of functions whose definition is motivated by the Blaschke Rolling Theorem. Given $0 \leq R_{1} \leq R_{2} \leq \infty$ and $R_{2} \geq 1$, let $\operatorname{CVG}\left(R_{1}, R_{2}\right)$ be the class of functions $f$ in $\mathbf{S}$ with the property that for each $\eta \in \partial f(\mathbf{D})$ there are open disks $D_{1}(\eta)$ and $D_{2}(\eta)$ of radius $R_{1}$ and $R_{2}$, respectively, such that $\eta \in \partial D_{1}(\eta) \cap \partial D_{2}(\eta)$ and

$$
D_{1}(\eta) \subseteq f(\mathbf{D}) \subseteq D_{2}(\eta) .
$$

If $R_{1}=0$ or $R_{2}=\infty$ we interpret $D_{1}(\eta)$ or $D_{2}(\eta)$ to be the empty set or an open half-plane, respectively.

If $f$ is a bounded function in $\operatorname{CVG}\left(R_{1}, R_{2}\right)$ and $R_{1}>0$, then $\partial f(\mathbf{D})$ is smooth in the sense that it has a continuously turning tangent. Yet we shall see that $\partial f(\mathbf{D})$ need not be a curve of class $C^{2}$ and $\rho(z)$ may not be continuous on $\overline{\mathbf{D}}$. In $\S 2$ we show that $\mathrm{CV}\left(R_{1}, R_{2}\right) \subseteq \mathrm{CVG}\left(R_{1}, R_{2}\right)$. Next, $\S 3$ concerns characterizations of $\operatorname{CVG}\left(R_{1}, R_{2}\right)$ in terms of the geometry of the regions $f(D)$. In $\S 4$ we obtain some partial results on the relationship between the largest disk centered at zero which is covered by $f(\mathbf{D})$ and the $\sup \{|f(z)|: z \in$ D $\}$ when $f \in \operatorname{CVG}\left(R_{1}, R_{2}\right)$. Finally, we study the order of growth of $\left|f^{\prime}\right|$ and discuss some open questions.

\section{Preliminaries}

Each $f$ in $\mathrm{CV}$ has a spherically continuous extension to $\overline{\mathbf{D}}$. Moreover, there are at most two points on $\partial \mathbf{D}$ at which $f$ takes the value infinity, and except for this occurrence, $f(\partial \mathbf{D})$ is a simple closed curve in the extended complex plane. Since $z f^{\prime}$ is starlike, $\arg z f^{\prime}(z)$ has a radial limit everywhere [6]. Thus, though $f(\partial \mathbf{D})$ may not be smooth, $n(\zeta)$ does exist as a radial limit for all $\zeta \in \partial \mathbf{D}$. Let

$$
\Phi(t)=\lim _{r \rightarrow 1} \arg n\left(r e^{i t}\right) .
$$

Since $\Phi$ is increasing [6], it has at most a countable number of discontinuities, each of which is a simple jump. Moreover, if $\Phi$ has a jump at $t$ of magnitude $\alpha \pi, \alpha>0$, and $f\left(e^{i t}\right)$ is finite, then $\alpha<1$, and we shall say that $\partial f(\mathbf{D})$ has a corner at $f\left(e^{i t}\right)$ with interior angle $(1-\alpha) \pi$.

Lemma 1. If $f \in \mathrm{CV}$ and $\partial f(\mathbf{D})$ has a corner at $f\left(e^{i t_{0}}\right)$, then $\rho\left(r e^{i t_{0}}\right) \rightarrow 0$ as $r \rightarrow 1$.

Proof. Assume $f \in \mathrm{CV}$. Pommerenke [6, Lemma 1] showed that

$$
z f^{\prime}(z)=z \exp \left\{(-1 / \pi) \int_{0}^{2 \pi} \log \left(1-z e^{-i t}\right) d \Phi(t)\right\}
$$


so

$$
\rho(z)=\left|z f^{\prime}(z)\right| /\left[(1 / 2 \pi) \int_{0}^{2 \pi} \operatorname{Re}\left\{\left(1+z e^{-i t}\right) /\left(1-z e^{-i t}\right)\right\} d \Phi(t)\right] .
$$

Suppose $\Phi$ has a jump of magnitude $\alpha \pi$ at $t_{0}$. If $\delta \in(0, \pi)$, then

$$
\begin{aligned}
(1 / 2 \pi) & \int_{0}^{2 \pi} \operatorname{Re}\left\{\left(1+r e^{i\left(t_{0}-t\right)}\right) /\left(1-r e^{i\left(t_{0}-t\right)}\right)\right\} d \Phi(t) \\
\geq & (1 / 2 \pi) \int_{t_{0}-\delta}^{t_{0}+\delta} \operatorname{Re}\left\{\left(1+r e^{i\left(t_{0}-t\right)}\right) /\left(1-r e^{i\left(t_{0}-t\right)}\right)\right\} d \Phi(t),
\end{aligned}
$$

and the quantity on the right decreases to $\alpha(1+r) / 2(1-r)$ as $\delta$ decreases to zero. Thus, for $z=r e^{i t_{0}}$,

$$
\operatorname{Re}\left\{1+z f^{\prime \prime}(z) / f^{\prime}(z)\right\} \geq \alpha(1+r) / 2(1-r) .
$$

Moreover, Pommerenke [6, p. 212] showed that if $\eta>0$ and $z=r e^{i t_{0}}$, then

$$
\ln \left|z f^{\prime}(z)\right|<\ln r+(1 / \pi)\left[\Phi\left(t_{0}+\eta\right)-\Phi\left(t_{0}-\eta\right)\right] \ln [(1+r) /(1-r)]+B,
$$

where $B=2 \pi / \sin ^{2}(\eta)$. Given $\varepsilon>0$, fix $\eta>0$ such that $\Phi\left(t_{0}+\eta\right)-\Phi\left(t_{0}-\eta\right)<$ $(\alpha+\varepsilon) \pi$. It then follows from (2) and (3) that

$$
\alpha \rho(z) \leq 2 r e^{B}[(1-r) /(1+r)]^{1-\alpha-\varepsilon} .
$$

Since $\alpha<1$, we may choose $\varepsilon$ so that $1-\alpha-\varepsilon>0$, and the conclusion follows.

Let $f_{r}(z)=f(r z) / r, 0<r<1$. Since $\rho\left(f_{r} ; z\right)$ is continuous on $\overline{\mathbf{D}}$, it is easy to see that $R_{1}\left(f_{r}\right)$ and $R_{2}\left(f_{r}\right)$ are the minimum and maximum, respectively, of $\rho\left(f_{r} ; z\right)$ on $\partial \mathbf{D}$. Then $R_{1}\left(f_{r}\right)=\rho_{m}(r) / r$ and $R_{2}\left(f_{r}\right)=\rho_{M}(r) / r$, so $R_{1}(f)$ and $R_{2}(f)$ are the limit inferior and limit, respectively, of $R_{1}\left(f_{r}\right)$ and $R_{2}\left(f_{r}\right)$, as $r \rightarrow 1$.

Theorem 1. $\mathrm{CV}\left(R_{1}, R_{2}\right) \subseteq \mathrm{CVG}\left(R_{1}, R_{2}\right)$.

Proof. Assume $f \in \mathrm{CV}\left(R_{1}, R_{2}\right)$, and let $\eta=f(\zeta) \in \partial f(\mathbf{D})$. Applying Blaschke's Rolling Theorem to $f_{r}$, we have

(4) $B\left(f_{r}(\zeta)-R_{1}\left(f_{r}\right) n(r \zeta), R_{1}\left(f_{r}\right)\right) \subseteq f_{r}(\mathbf{D}) \subseteq B\left(f_{r}(\zeta)-R_{2}\left(f_{r}\right) n(r \zeta), R_{2}\left(f_{r}\right)\right)$

for each $\zeta \in \partial \mathbf{D}$. If $w \in f(\mathbf{D})$, then $w \in f_{r}(\mathbf{D})$ for sufficiently large $r$. Letting $r$ tend to 1 , the right side of (4) yields

$$
w \in \overline{B\left(f(\zeta)-R_{2}(f) n(\zeta), R_{2}(f)\right)} \subseteq \overline{B\left(f(\zeta)-R_{2} n(\zeta), R_{2}\right)}
$$

Setting $D_{2}(\eta)=B\left(f(\zeta)-R_{2} n(\zeta), R_{2}\right)$, we conclude (since $f(\mathbf{D})$ is open) that $f(\mathbf{D}) \subseteq D_{2}(\eta)$. This establishes the right side of (1). If $R_{1}=0$, then the left side of (1) is clear, so assume $R_{1}>0$. Let $w \in B\left(f(\zeta)-R_{1}(f) n(\zeta), R_{1}(f)\right)$ and choose a sequence of values of $r$ converging to 1 such that $R_{1}\left(f_{r}\right) \rightarrow R_{1}(f)$. From (4) we have

$$
w \in B\left(f_{r}(\zeta)-R_{1}\left(f_{r}\right) n(r \zeta), R_{1}\left(f_{r}\right)\right) \subseteq f_{r}(\mathbf{D})
$$


for all these values of $r$ which are sufficiently near 1 , and consequently $w \in$ $f(\mathbf{D})$. Thus,

$$
B\left(f(\zeta)-R_{1} n(\zeta), R_{1}\right) \subseteq B\left(f(\zeta)-R_{1}(f) n(\zeta), R_{1}(f)\right) \subseteq f(\mathbf{D}),
$$

and $D_{1}(\eta)=B\left(f(\zeta)-R_{1} n(\zeta), R_{1}\right)$ satisfies the left side of (1).

Suppose $\Omega$ is a bounded convex region. Given $z \in \Omega$, let $\phi_{z}$ denote the conformal mapping of $\mathbf{D}$ onto $\Omega$ satisfying $\phi_{z}(0)=z$ and $\phi_{z}^{\prime}(0)>0$. The hyperbolic metric for $\Omega$ has density $\lambda_{\Omega}(z)=1 / \phi_{z}^{\prime}(0)$. The following lemma will be needed in subsequent sections and follows directly from results on $\lambda_{\Omega}$ in $[5$, p. 474].

Lemma 2. Suppose $\Omega$ is a bounded convex region that is symmetric about two distinct lines which intersect at $z_{0}$. If $z_{1} \in \Omega \backslash\left\{z_{0}\right\}$ and $z_{t}=(1-t) z_{0}+t z_{1}$, $0<t<1$, then $\phi_{z_{1}}^{\prime}(0)<\phi_{z_{t}}^{\prime}(0)<\phi_{z_{0}}^{\prime}(0)$.

\section{Geometry of $f(\mathbf{D})$}

Assume $0<R<\infty, a, b \in \mathbf{C}$ (the complex numbers), and $|a-b| \leq 2 R$. If $|a-b|<2 R$, then there are two open disks $\Delta_{1}$ and $\Delta_{2}$ of radius $R$ such that $a, b \in \partial \Delta_{1} \cap \partial \Delta_{2}$, and we set $E(a, b ; R)=\Delta_{1} \cap \Delta_{2}$. If $|a-b|=2 R$, set $E(a, b ; R)=B((a+b) / 2, R)$. We shall say that a set $A \subseteq \mathbf{C}$ is $R$-convex if $\operatorname{diam}(A) \leq 2 R$ and $E(a, b ; R) \subseteq A$ for all choices of $a$ and $b$ in $A$. Assuming $\operatorname{diam}(A) \leq 2 R$, we define the $R$-hull of $A$ to be the smallest $R$ convex set containing $A$ and denote it by $\operatorname{co}_{R}(A)$. In the limiting case when $R=\infty$ we set $E(a, b ; \infty)=(a, b)$, which yields the usual notions of convexity and convex hull. Mejia and Minda [4] obtained the following result.

Theorem A. If $1 \leq R \leq \infty$ and $f \in \mathbf{S}$, then $f \in \mathrm{CV}(0, R)$ if and only if $\operatorname{diam} f(\mathbf{D}) \leq 2 R$ and $E(a, b ; R) \subseteq f(\mathbf{D})$ for all $a, b \in f(\mathbf{D})$.

In the theorem which follows we present characterizations of $\operatorname{CVG}\left(R_{1}, R_{2}\right)$ in terms of the geometry of $f(\mathbf{D})$. Suppose $0<R_{1} \leq R_{2} \leq \infty$ and let $a, b \in \mathbf{C}$. If $|a-b| \leq 2\left(R_{2}-R_{1}\right)$, then $\operatorname{diam}\left(B\left(a, R_{1}\right) \cup B\left(b, R_{1}\right)\right) \leq 2 R_{2}$, and we set $E\left(a, b ; R_{1}, R_{2}\right)=\operatorname{co}_{R_{2}}\left(B\left(a, R_{1}\right) \cup B\left(b, R_{1}\right)\right)$. If $R_{1}=0$ and $R_{2}>0$, we set $E\left(a, b ; 0, R_{2}\right)=E\left(a, b ; R_{2}\right)$.

Theorem 2. If $0 \leq R_{1} \leq R_{2} \leq \infty, R_{2} \geq 1$, and $f \in \mathbf{S}$, then the following are equivalent:

(i) $f \in \mathrm{CVG}\left(R_{1}, R_{2}\right)$;

(ii) $f(\mathbf{D})$ is the intersection of open disks of radius $R_{2}$ and, in the case $R_{1}>0$, the union of open disks of radius $R_{1}$;

(iii) $\forall u, v \in f(\mathbf{D}) \exists a, b \in f(\mathbf{D})$ such that $u, v \in E\left(a, b ; R_{1}, R_{2}\right) \subseteq f(\mathbf{D})$;

(iv) $f \in \mathbf{C V}$, and for each $\zeta \in \partial \mathbf{D}$ for which $f(\zeta)$ is finite,

$$
f(\mathbf{D}) \subseteq B\left(f(\zeta)-R_{2} n(\zeta), R_{2}\right)
$$

and in the case $R_{1}>0, B\left(f(\zeta)-R_{1} n(\zeta), R_{1}\right) \subseteq f(\mathbf{D})$. 
Remark. If $R_{2}=\infty$, then an open disk of radius $R_{2}$ is to be interpreted as an open half-plane, and $B\left(f(\zeta)-R_{2} n(\zeta), R_{2}\right)$ is to be interpreted as the limiting half-plane as $R_{2} \rightarrow \infty$.

Proof. (i) $\Rightarrow$ (ii). Assuming $f \in \operatorname{CVG}\left(R_{1}, R_{2}\right)$, we assert that $f(\mathbf{D})=$ $\bigcap\left\{D_{2}(\eta): \eta \in \partial f(\mathbf{D})\right\}$. Certainly $f(\mathbf{D}) \subseteq \bigcap\left\{D_{2}(\eta): \eta \in \partial f(\mathbf{D})\right\}$. To obtain the opposite containment, suppose $w \notin f(\mathbf{D})$. Fix $w_{0} \in f(\mathbf{D})$ and choose $\eta \in\left[w_{0}, w\right] \cap \partial f(\mathbf{D})$. Then $f(\mathbf{D}) \subseteq D_{2}(\eta)$ implies $w \notin D_{2}(\eta)$, and thus $w \notin \bigcap\left\{D_{2}(\eta): \eta \in \partial f(\mathbf{D})\right\}$. Now assume $R_{1}>0$ and let $w \in f(\mathbf{D})$. If $\operatorname{dist}(w, \partial f(\mathbf{D})) \geq R_{1}$, let $D(w)=B\left(w, R_{1}\right)$. If $\left.\operatorname{dist}(w, \partial f \mathbf{D})\right)<R_{1}$, choose $\eta \in \partial(f(\mathbf{D}))$ such that $|\eta-w|=\operatorname{dist}(w, \partial f(\mathbf{D}))$ and let $D(w)=D_{1}(\eta)$. Then $f(\mathbf{D})=\bigcup\{D(w): w \in f(\mathbf{D})\}$, and (ii) is established.

(ii) $\Rightarrow$ (iii). Let $u, v \in f(\mathbf{D})$. Suppose first that $R_{1}=0$ and let $\left\{D_{\alpha}: \alpha \in\right.$ $A\}$ be a collection of open disks of radius $R_{2}$ such that $f(\mathbf{D})=\bigcap\left\{D_{\alpha}: \alpha \in A\right\}$. Since $f(\mathbf{D})$ is convex (being the intersection of open disks), $[u, v] \subseteq f(\mathbf{D})$. Choose $a$ and $b$ on the line determined by $u$ and $v$ such that $[u, v] \subseteq$ $(a, b) \subseteq f(\mathbf{D})$. For each $\alpha \in A,(a, b) \subseteq D_{\alpha}$, and hence $E\left(a, b ; R_{2}\right) \subseteq D_{\alpha}$. Thus, $u, v \in E\left(a, b ; R_{2}\right) \subseteq f(\mathbf{D})$. Now assume that $R_{1}>0$, in which case we assume in addition that $f(\mathbf{D})$ is the union of open disks of radius $R_{1}$. Choose open disks $D_{u}$ and $D_{v}$ of radius $R_{1}$ with centers $a$ and $b$, respectively, such that $u \in D_{u} \subseteq f(\mathbf{D})$ and $v \in D_{v} \subseteq f(\mathbf{D})$. Since $f(\mathbf{D})$ lies in a disk of radius $R_{2}, \operatorname{diam}\left(D_{u} \cup D_{v}\right) \leq 2 R_{2}$. We claim that $E\left(a, b ; R_{1}, R_{2}\right)=\operatorname{co}_{R_{2}}\left(D_{u} \cup D_{v}\right) \subseteq$ $f(D)$. Indeed, since $\operatorname{CVG}\left(R_{1}, R_{2}\right) \subseteq \operatorname{CVG}\left(0, R_{2}\right)$, we may apply the earlier part of the proof to conclude that $E\left(a^{\prime}, b^{\prime} ; R_{2}\right) \subseteq f(\mathbf{D})$ for all $a^{\prime} \in D_{u}$ and $b^{\prime} \in D_{v}$, and the conclusion follows.

(iii) $\Rightarrow$ (iv). Since $E\left(a, b ; R_{1}, R_{2}\right)$ is a convex set, (iii) implies that $f(\mathbf{D})$ is convex. It is implicit in (iii) that $\operatorname{diam} f(\mathbf{D}) \leq 2 R_{2}$. Fix $\zeta \in \partial \mathbf{D}$ such that $f(\zeta) \neq \infty$, and consider $w \in f(\mathbf{D})$. Suppose first that $R_{1}>0$. For sufficiently small $\varepsilon>0, f(\zeta)-\varepsilon n(\zeta) \in f(\mathbf{D})$, so there are points $a_{\varepsilon}$ and $b_{\varepsilon}$ such that $w, f(\zeta)-\varepsilon n(\zeta) \in E\left(a_{\varepsilon}, b_{\varepsilon} ; R_{1}, R_{2}\right) \subseteq f(\mathbf{D})$. Let $\varepsilon$ tend to zero though a sequence of values for which $a_{\varepsilon}$ and $b_{\varepsilon}$ are convergent, say to $a$ and $b$, respectively. Then $w \in \overline{E\left(a, b ; R_{1}, R_{2}\right)}$, and $f(\zeta) \in \partial E\left(a, b ; R_{1}, R_{2}\right)$. Since $R_{1}>0, \partial f(\mathbf{D})$ has a tangent at $f(\zeta)$, and it follows from the shape of $E\left(a, b ; R_{1}, R_{2}\right)$ that

$$
B\left(f(\zeta)-R_{1} n(\zeta), R_{1}\right) \subseteq E\left(a, b ; R_{1}, R_{2}\right) \subseteq B\left(f(\zeta)-R_{2} n(\zeta), R_{2}\right)
$$

Thus, $B\left(f(\zeta)-R_{1} n(\zeta), R_{1}\right) \subseteq f(\mathbf{D})$, and $w \in \overline{B\left(f(\zeta)-R_{2} n(\zeta), R_{2}\right)}$ for all $w \in f(\mathbf{D})$. Since $f(\mathbf{D})$ is open we conclude that $f(\mathbf{D}) \subseteq B\left(f(\zeta)-R_{2} n(\zeta), R_{2}\right)$. Now consider the case $R_{1}=0$. The preceding argument yields $f(\mathbf{D}) \subseteq$ $B\left(f(\zeta)-R_{2} n(\zeta), R_{2}\right)$ when $\partial f(\mathbf{D})$ has a tangent at $f(\zeta)$, and this occurs for all but a countable number of points $\zeta \in \partial \mathbf{D}$. Suppose $\partial f(\mathbf{D})$ has a corner at $f(\zeta), \zeta=e^{i \theta}$, and let $n\left(\zeta^{+}\right)$and $n\left(\zeta^{-}\right)$denote the limits of $n\left(e^{i t}\right)$ as $t$ tends to $\theta$ from the right and left, respectively. From the previous case we 
conclude that

$$
f(\mathbf{D}) \subseteq B\left(f(\zeta)-R_{2} n\left(\zeta^{+}\right), R_{2}\right) \cap B\left(f(\zeta)-R_{2} n\left(\zeta^{-}\right), R_{2}\right) .
$$

Since, $\arg n\left(\zeta^{-}\right) \leq \arg n(\zeta) \leq \arg n\left(\zeta^{+}\right)$, the right side of Equation (5) lies in $B\left(f(\zeta)-R_{2} n(\zeta), R_{2}\right)$.

Finally, it is clear that (iv) implies (i).

The following result occurs in the proof of (iii) implies (iv) in Theorem 2, and we record it here for emphasis.

Corollary. Suppose $f \in \operatorname{CVG}\left(0, R_{2}\right)$ and $\partial f(\mathbf{D})$ has a corner at $f(\zeta), \zeta=$ $e^{i t_{0}}$. Then

$$
f(\mathbf{D}) \subseteq B\left(f(\zeta)-R_{2} n\left(\zeta^{+}\right), R_{2}\right) \cap B\left(f(\zeta)-R_{2} n\left(\zeta^{-}\right), R_{2}\right),
$$

where $n\left(\zeta^{+}\right)$and $n\left(\zeta^{-}\right)$denote the limits of $n\left(e^{i t}\right)$ as $t \rightarrow t_{0}$ from the right and left, respectively.

\section{COVERING PROPERTIES}

Given $f$ in $\mathrm{CV}$, let $d(f)$ and $M(f)$ be the minimum and maximum, respectively, of $|f(z)|$ on $|z|=1$. In general, $1 / 2 \leq d(f) \leq M(f) \leq \infty$. Goodman [1] considered the problems of finding inf $d(f)$ and $\sup M(f)$ for $f$ in $\operatorname{CV}(0, R)$, and he found the following function and its rotations to be extremal for both problems.

Example. If $1 \leq R<\infty$, let $k_{R}(z)=z /(1-z \sqrt{1-1 / R})$, and if $R=\infty$, let $k_{\infty}(z)=z /(1-z)$. Then $k_{R}(\mathbf{D})$ is $B\left(\sqrt{R^{2}-R}, R\right)$ or $\{z: \operatorname{Re} z>-1 / 2\}$, depending on whether $R<\infty$ or $R=\infty$, respectively. To avoid the need to distinguish between the two cases, $R<\infty$ and $R=\infty$, we shall agree, in the case $R=\infty$, to interpret $B\left(\sqrt{R^{2}-R}, R\right)$ to be $\{z: \operatorname{Re} z>1 / 2\}$ and quantities such as $R-\sqrt{R^{2}-R}$ to be their limits (in this case $1 / 2$ ) as $R \rightarrow \infty$. Then for $1 \leq R \leq \infty$ we have $R_{1}\left(k_{R}\right)=R=R_{2}\left(k_{R}\right), d\left(k_{R}\right)=R-\sqrt{R^{2}-R}$, and $M\left(k_{R}\right)=R+\sqrt{R^{2}-R}$.

The conclusions of the following theorem were obtained by Goodman [1] for the class $\operatorname{CV}\left(R_{1}, R_{2}\right)$. The proof for $\operatorname{CVG}\left(R_{1}, R_{2}\right)$ is virtually the same and will not be repeated.

Theorem B. If $f \in \mathrm{CVG}\left(R_{1}, R_{2}\right)$, then

$$
d(f) \geq R_{2}-\sqrt{R_{2}^{2}-R_{2}}
$$

and

$$
M(f) \leq R_{2}+\sqrt{R_{2}^{2}-R_{2}}
$$


Equality occurs in (6) or (7) if and only if $f$ is a rotation of $k_{R_{2}}$. Moreover, if $1 \leq R_{1} \leq \infty$, then

$$
d(f) \leq R_{1}-\sqrt{R_{1}^{2}-R_{1}},
$$

with equality if and only if $f$ is a rotation of $k_{R_{1}}$.

We now look at the effect on $M(f)$ when $f \in \mathrm{CVG}\left(R_{1}, R_{2}\right)$ and $f$ covers the disk $B(0, d)$, where $R_{2}-\sqrt{R_{2}^{2}-R_{2}}<d<1$. We shall need to also assume that $R_{1} \leq d$. The case $R_{2}=\infty$ is somewhat special in that functions in $\operatorname{CVG}\left(R_{1}, \infty\right)$ need not be bounded, and we begin by discussing some of the features of this situation.

Given $r>0$, let $g_{r}$ be the conformal mapping of $\mathbf{D}$ onto $\operatorname{co}(B(0, r) \cup$ $(0, \infty))$ such that $g_{r}(0)=0$ and $g_{r}^{\prime}(0)>0$. If $0<r_{1}<r_{2}$, then $g_{r_{1}}$ is properly subordinate to $g_{r_{2}}$, and consequently $g_{r}^{\prime}(0)$ is a strictly increasing function of $r$. If $r \leq \pi / 4$, then $g_{r} \prec(1 / 2) \log [(1+z) /(1-z)]$ and $g_{r}^{\prime}(0)<1$, whereas $r \geq 1$ implies $z \prec g_{r}$ and $g_{r}^{\prime}(0)>1$. Thus, there is a unique $r^{*} \in(\pi / 4,1)$ such that $g^{*}=g_{r^{*}} \in \mathrm{CV}$.

Lemma 3. Assume $f \in \mathrm{CV}$. If $d(f)>r^{*}$, then $f$ is bounded. If $d(f)=r^{*}$ and $f$ is not bounded, then $f$ is a rotation of $g^{*}$.

Proof. Suppose $d(f) \geq r^{*}$ and $f$ is not bounded. Choose $\left\{w_{n}\right\}_{1}^{\infty} \subseteq f(\mathbf{D})$ such that $w_{n} \rightarrow \infty$ and $\arg w_{n} \rightarrow \beta$. By a rotation we need only consider $\beta=0$, in which case $\operatorname{co}(B(0, d(f)) \cup(0, \infty)) \subseteq f(\mathbf{D})$. Thus, $g^{*} \prec f$, and, since both functions are normalized, we conclude that $g^{*}=f$ and $d(f)=r^{*}$.

Now, suppose $0 \leq R_{1} \leq R_{2}<\infty, R_{2}>1, R_{2}-\sqrt{R_{2}^{2}-R_{2}}<d<1$, and $R_{1} \leq d$. Let $a \in\left[R_{1}-d, 2 R_{2}-R_{1}-d\right]$ and let $U_{a}$ be $B(0, d) \cup$ $B\left(a, R_{1}\right)$ or $B(0, d) \cup(0, a)$ depending on whether $R_{1}>0$ or $R_{1}=0$. Then diam $U_{a} \leq 2 R_{2}$, and there is a unique conformal map $\varphi_{a}$ of $\mathbf{D}$ onto $\mathrm{co}_{R_{2}}\left(U_{a}\right)$ such that $\varphi_{a}(0)=0$ and $\varphi_{a}^{\prime}(0)>0$. If $a \in\left[R_{1}-d, d-R_{1}\right]$, then $\varphi_{a}(\mathbf{D})=B(0, d)$ and $\varphi_{a}^{\prime}(0)=d<1$. Since $R_{1} \leq d$, the regions $\varphi_{a}(\mathbf{D})$ expand as $a$ increases, and consequently $\varphi_{a}^{\prime}(0)$ is a strictly increasing function of $a$ on $\left[d-R_{1}, 2 R_{2}-R_{1}-d\right]$. If $a=2 R_{2}-R_{1}-d$, then $\varphi_{a}(\mathbf{D})=B\left(R_{2}-d, R_{2}\right)$, and since $d>R_{2}-\sqrt{R_{2}^{2}-R_{2}}$, Lemma 2 implies that $\varphi_{a}^{\prime}(0)>k_{R_{2}}^{\prime}(0)=1$. Thus, there is a unique $a \in\left[d-R_{1}, 2 R_{2}-R_{1}-d\right]$ for which $\varphi_{a}^{\prime}(0)=1$. This normalized function, denoted hereafter by $h_{d}$, is in $\operatorname{CVG}\left(R_{1}, R_{2}\right)$.

Now, consider $R_{2}=\infty$. In this case we choose $a \in\left[R_{1}-d, \infty\right)$ and define $\varphi_{a}$ as before. As long as $d>r^{*}, \lim _{a \rightarrow \infty} \varphi_{a}^{\prime}(0)=g_{d}^{\prime}(0)>1$, and again there will be a unique value of $a$ for which $\varphi_{a}^{\prime}(0)=1$. This normalized function will also be denoted by $h_{d}$.

Theorem 3. Assume $0 \leq R_{1} \leq d, R_{2}>1$, and $R_{2}-\sqrt{R_{2}^{2}-R_{2}}<d<1$. Let $f \in \operatorname{CVG}\left(R_{1}, R_{2}\right)$ and suppose $d(f) \geq d$. If $R_{2}<\infty$, then $M(f) \leq M\left(h_{d}\right)$, 
with equality if and only if $f$ is a rotation of $h_{d}$. If $R_{2}=\infty$, then the same conclusions hold as long as $d>r^{*}$.

Proof. In either case, choose $\zeta \in \partial \mathbf{D}$ such that $|f(\zeta)|=M(f)$. By a rotation it suffices to consider $f(\zeta)>0$, in which case $n(\zeta)=1$. Then,

$$
B\left(f(\zeta)-R_{1}, R_{1}\right) \subseteq f(\mathbf{D}),
$$

and consequently $\mathrm{co}_{R_{2}}\left(B(0, d) \cup B\left(f(\zeta)-R_{1}, R_{1}\right)\right) \subseteq f(\mathbf{D})$. If $f(\zeta) \geq M\left(h_{d}\right)$, then $h_{d}$ is subordinate to $f$. But, $f^{\prime}(0)=1=h_{d}^{\prime}(0)$, so $f=h_{d}$.

If $R_{2}=\infty, 1 / 2 \leq d \leq r^{*}$, and $f \in \operatorname{CVG}\left(R_{1}, R_{2}\right)$, then $f(\mathbf{D})$ may not be bounded, but there is a restriction on how large a sector $f$ may cover. For each $\theta \in(0, \pi]$, let $\sigma_{\theta}$ be the conformal map of $\mathbf{D}$ onto $\operatorname{co}(B(0, d) \cup\{z:|\arg z|<$ $\theta / 2\})$ such that $\sigma_{\theta}(0)=0$ and $\sigma_{\theta}^{\prime}(0)>0$. Let $\sigma_{0}=g_{d}$. Then $\sigma_{\theta}^{\prime}(0)$ is a strictly increasing function of $\theta$ on $[0, \pi]$. Moreover, $d \geq 1 / 2$ implies $z /(1-z) \prec \sigma_{\pi}$ and $\sigma_{\pi}^{\prime}(0) \geq 1$, with equality only when $d=1 / 2$. Also, $d \leq r^{*}$ implies $\sigma_{0} \prec g^{*}$ and $\sigma_{0}^{\prime}(0) \leq 1$, with equality only when $d=r^{*}$. Thus, there is a unique $\theta(d)$ in $[0, \pi]$ such that $\sigma_{\theta(d)}^{\prime}(0)=1$, and we denote this function by $s_{d}$.

Theorem 4. Suppose $1 / 2 \leq d<r^{*}, f \in \mathrm{CVG}\left(R_{1}, \infty\right), R_{1} \leq d$, and $d(f) \geq d$. If $f(\mathbf{D})$ contains an open sector of angle $\theta(d)$, then $f$ is a rotation of $s_{d}$.

Proof. Suppose $f(\mathbf{D})$ contains an open sector $\Sigma$ with vertex angle $\theta(d)$. By a rotation we may assume that $\Sigma$ is symmetric with respect to the real axis and contains $(0, \infty)$, in which case $s_{d} \prec f$. Thus, $f=s_{d}$.

\section{GROWTH OF $\left|f^{\prime}\right|$}

The following result, due to Pommerenke [6, Theorem 1], shows that for convex functions the growth of $M\left(r, f^{\prime}\right)=\max \left\{\left|f^{\prime}(z)\right|:|z|=r\right\}$ is governed by the magnitude of the largest jump of $\Phi$.

Theorem C. If $f \in \mathrm{CV}$ and $\alpha \pi=\max \left\{\boldsymbol{\Phi}\left(t^{+}\right)-\Phi\left(t^{-}\right): 0 \leq t \leq 2 \pi\right\}$, then

$$
M\left(r, f^{\prime}\right) \geq 1 / 4(1-r)^{\alpha}
$$

and

$$
\alpha=\lim _{r \rightarrow 1} \log M\left(r, f^{\prime}\right) / \log (1 /(1-r)) .
$$

If $f \in \operatorname{CVG}\left(R_{1}, R_{2}\right), f(\mathbf{D})$ is bounded, and $R_{1}>0$, then $\Phi$ is continuous and $\alpha=0$. Hence we consider the case $R_{1}=0, R_{2}=R \in[1, \infty)$, and proceed to determine the largest possible jump of $\Phi$. First we discuss the function in $\operatorname{CVG}(0, R)$ which exhibits the extremal behavior.

Example. Suppose $\alpha \in[0,1)$, let $\phi(z)=(1+z) /(1-z)$, and consider $f_{\alpha}(z)=$ $\phi^{-1}\left(\phi(z)^{1-\alpha}\right) /(1-\alpha)$. Elementary calculations show that $f(\mathbf{D})=E(a, b ; R)$, where $a=-(1-\alpha)^{-1}, b=(1-\alpha)^{-1}$, and

$$
R=1 /(1-\alpha) \sin [(1-\alpha) \pi / 2] \text {. }
$$


Thus, $f \in \operatorname{CVG}(0, R)$. For each $R \in[1, \infty)$ there is a unique $\alpha=\alpha(R) \in$ $[0,1)$ determined implicitly by $(11)$, and wet set $f_{R}=f_{\alpha(R)}$. The function $\Phi\left(f_{R}, t\right)$ is continuous on $(0, \pi) \cup(\pi, 2 \pi)$ and has a jump discontinuity of magnitude $\alpha(R) \pi$ at 0 and at $\pi$.

Theorem 5. If $1 \leq R<\infty$ and $f \in \operatorname{CVG}(0, R)$, then $\Phi\left(t^{+}\right)-\Phi\left(t^{-}\right) \leq \alpha(R) \pi$ for all $t$, and equality occurs for some $t$ if and only if $f$ is a rotation of $f_{R}$.

Proof. Fix $\zeta=e^{i t}$ and assume $\Phi\left(t^{+}\right)-\Phi\left(t^{-}\right)=\beta \pi>0$. By the corollary to Theorem 2,

$$
f(\mathbf{D}) \subseteq \Omega=B\left(f(\zeta)-R n\left(\zeta^{+}\right), R\right) \cap B\left(f(\zeta)-R n\left(\zeta^{-}\right), R\right) .
$$

Note that $\Omega=E(f(\zeta), b ; R)$ for an appropriate choice of $b \in \mathbf{C}$, and the interior angle of $\Omega$ at $f(\zeta)$ is $(1-\beta) \pi$. There exist $\mu, \nu \in \mathbf{C},|\mu|=1$, such that $G=\left(\mu f_{R}+\nu\right)(\mathbf{D})=E(f(\zeta), c ; R)$, where $c$ lies on the ray from $f(\zeta)$ through $b$. Now suppose $\beta>\alpha(R)$. Then $|c-f(\zeta)|>|b-f(\zeta)|$ and $f(\mathbf{D}) \subseteq \Omega \subseteq G$. Let $g$ be the conformal map of $\mathbf{D}$ onto $G$ such that $g(0)=f(0)$ and $g^{\prime}(0)>0$. Then $\beta>\alpha(R)$ implies $f$ is properly subordinate to $g$, and consequently $1=f^{\prime}(0)<g^{\prime}(0)$. But Lemma 2 gives $g^{\prime}(0) \leq$ $\left|\left(\mu f_{R}+\nu\right)^{\prime}(0)\right|=1$, a contradiction. Thus, $\beta \leq \alpha(R)$, and the conclusion follows. If $\beta=\alpha(R)$, then the same considerations yield $f(\mathbf{D})=G$ and $f(0)=0=\left(\mu f_{R}+\nu\right)(0)$, so $f$ is a rotation of $f_{R}$.

Corollary. If $1 \leq R<\infty$ and $f \in \operatorname{CVG}(0, R)$, then

$$
M\left(r, f^{\prime}\right)=O\left((1-r)^{-\alpha(R)}\right) .
$$

Proof. Suppose $f \in \operatorname{CVG}(0, R)$ and $f \neq f_{R}$. By the previous theorem, $\beta=$ $\max \left\{\boldsymbol{\Phi}\left(t^{+}\right)-\Phi\left(t^{-}\right): 0 \leq t \leq 2 \pi\right\} / \pi<\alpha(R)$. If $\varepsilon$ is chosen so that $\beta+\varepsilon<\alpha(R)$, then (10) gives $M\left(r, f^{\prime}\right)=O\left((1-r)^{-(\beta+\varepsilon)}\right)=o\left((1-r)^{-\alpha(R)}\right)$. Now, consider $f_{R}$. A brief calculation yields

$$
f_{R}^{\prime}(z)=4 /\left[(1+z)^{1-\alpha(R)}+(1-z)^{1-\alpha(R)}\right]^{2}\left(1-z^{2}\right)^{\alpha(R)} .
$$

The quantity $(1+z)^{1-\alpha(R)}+(1-z)^{1-\alpha(R)}$ has a positive minimum modulus $m$ on $\overline{\mathbf{D}}$, so $\left|f_{R}^{\prime}(z)\right| \leq 4 / m^{2}\left(1-|z|^{2}\right)^{\alpha(R)}$, and hence $M\left(r, f_{R}^{\prime}\right)=O\left((1-r)^{-\alpha(R)}\right)$.

\section{Comments}

It follows readily from classical results in differential geometry (e.g., see [7]) that if $f \in \operatorname{CVG}\left(R_{1}, R_{2}\right)$ and $f$ is analytic on $\overline{\mathbf{D}}$, then $f \in \mathrm{CV}\left(R_{1}, R_{2}\right)$, but it remains to be determined whether or not $\operatorname{CVG}\left(R_{1}, R_{2}\right)=\operatorname{CV}\left(R_{1}, R_{2}\right)$. Mejia and Minda [4] have shown that this is the case when $R_{1}=0$, and we expect that it is also true for $R_{1}>0$.

Very little seems to be known about the behavior of $\rho_{m}(r)$ as $r \rightarrow 1$. Although Walsh noted that it need not be monotone, it would be interesting to know if $\rho_{m}(r)$ has a limit as $r \rightarrow 1$, or if, perhaps, it has at most a finite number of local extrema on $(0,1)$. 
The results in Theorem 3 are incomplete due to the assumption $R_{1} \leq d$. If $R_{1}>d$, then for an appropriate choice of $a \in\left[R_{1}-d, 2 R_{2}-R_{1}-d\right]$ there is a normalized conformal mapping of $\mathbf{D}$ onto $\mathrm{co}_{R_{2}}\left(B\left(R_{1}-d, R_{1}\right) \cup B\left(a, R_{1}\right)\right)$, and this may be the extremal function. However, in this case we do not get the subordination used in the proof of Theorem 3 because when $f$ is rotated so that $M(f)=f(\zeta)>0$, the point on $\partial f(\mathbf{D})$ nearest to $z=0$ may not be $-d$.

\section{REFERENCES}

1. A. W. Goodman, Convex functions of bounded type, Proc. Amer. Math. Soc. 92 (1984), 541-546.

2. __ More on convex functions of bounded type, Proc. Amer. Math. Soc. 97 (1986), 303306.

3. D. Koutroufiotis, On Blaschke's rolling theorems, Archiv der Math. 23 (1972), 655-660.

4. D. Mejia and D. Minda, Hyperbolic geometry in k-convex regions, Pacific J. Math. 141 (1990), 333-354.

5. C. D. Minda and D. J. Wright, Univalence criteria and the hyperbolic metric, Rocky Mountain J. Math. 12 (1982), 471-479.

6. Ch. Pommerenke, On starlike and convex functions, J. London Math. Soc. 37 (1962), 209224.

7. M. Spivak, A comprehensive introduction to differential geometry, vol. 2, Publish or Perish, Boston, 1970.

8. J. L. Walsh, Note on the curvature of level curves of Green's function, Proc. Nat. Acad. Sci. 23 (1937), 84-89.

9. K. L. Wirths, Coefficient bounds for convex functions of bounded type, Proc. Amer. Math. Soc. 103 (1988), 525-530.

Department of Mathematical Sciences, University of Cincinnati, Cincinnati, Ohio 45221 